\title{
A New Method for Electrostatic Ion Deflection
}

\author{
Barbara Wolf, Paul D. Mudgett, and Ronald D. Macfarlane \\ Department of Chemistry, Texas A\&M University, College Station, Texas, USA.
}

\begin{abstract}
A new approach to electrostatic ion deflection is described where an electrostatic particle guide (EPG) operating with reversed polarity is used to deflect ions in a cylindrical geometry about the axis of a time-of-flight mass spectrometer. The method has advantages over the standard parallel-plate deflector geometry in that it is more effective in deflecting ion beams that have a significant radial velocity component. The device is being used in ${ }^{252} \mathrm{Cf}$ plasma desorption mass spectrometry ( ${ }^{252} \mathrm{Cf}$-PDMS) experiments in an on/off mode to record neutral particle spectra and in a synchronized pulsed mode to reduce the magnitude of the uncorrelated background in the time-of-flight spectrum. Radial distribution functions have been measured for various EPG voltages. Its use as a background suppression technique is demonstrated by using the ${ }^{252} \mathrm{Cf}-\mathrm{PDMS}$ spectrum of insulin. (I Am Soc Mass Spectrom 1990, $1,28-36$ )
\end{abstract}

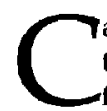
Talifornium-252 plasma desorption mass spectrometry $\left({ }^{252} \mathrm{Cf}-\mathrm{PDMS}\right)$ is a successful method for the detection of involatile high molecular weight biomolecules [1]. It has been widely used to analyze proteins of molecular weights up to 24,000 , and recently quasi-molecular ions of $m / z \sim 34,000$ were detected [2]. The sensitivity of the method for high-mass studies has been limited by a problem of resolving a high-intensity background from the relatively weak-intensity high-mass ions. Unlike conventional mass spectra, PDMS spectra typically contain peaks with broad and complex components superimposed on an extensive smoothly varying background. Chait and Field [3] studied the fragmentation process in PDMS by using retarding potentials to separate various components that may contribute to a single peak in the spectra. They found that metastable dissociation of desorbed ions is responsible for much of the background in PDMS. These processes create fragment ions and neutral fragments that contribute either to fragment ion peaks or to background in the spectrum depending on the site of the dissociation in the spectrometer. Metastable decays that occur in the field-free region of the spectromeler contribute to the broadening of the quasi-molecular ion peak. Internal energy is released as kinetic energy during fragmentation, and as a result the fragments have a distribution of velocities about the velocity of the precursor (quasi-molecular) ion. Sundqvist and co-workers [4] estimated that neutral fragments and neutralized parent ions from metastable decay contribute on the order

Address reprint requests to Ronald D. Macfarlane, Department of Chemistry. Texas A\&M University, College Station, TX 77843 . of $20 \%$ to a typical PDMS spectrum. The reduction of background that contributes to quasi-molecular ion peak breadth would improve the resolution of ${ }^{252} \mathrm{Cf}$ PDMS for higher molecular weight molecules as well as the sensitivity for detection.

Electrostatic particle guides (EPG) have been used in ${ }^{252} \mathrm{Cf}-\mathrm{PDMS}$ as a device for efficiently transmitting divergent ion beams over large distances [3,5]. The EPG was used by Oakey and Macfarlane [6] in 1967 as a means of transporting tritons, $\alpha$ particles, and fission fragments over a distance of $8 \mathrm{~m}$. The basic principle of operation of the device is that ions are injected into an electric field generated by two concentric cylinders. The inner cylinder is a fine wire at a potential that is attractive for the injected ion. A balance between electrostatic attraction and angular momentum conservation results in a spiral ion trajectory as the ion traverses the tube. More recently, the EPG has been used in ${ }^{252} \mathrm{Cf}$ PDMS to transmit low-energy $(5-80 \mathrm{eV})$ ions with high efficiency [7].

In this paper we describe the use of an EPG in a reverse mode as a means of ion rejection rather than transmission. The purpose is to study the neutral component of $a^{252} \mathrm{Cf}$-PDMS spectrum and resolve the neutral component from the ion spectrum. In a reverse mode the voltage applied to the center wire of the EPG is of the same polarity as the ions being detected, so ions are repelled but neutral fragments are unaffected. In the past, electrostatic deflector plates were used to reject unwanted ions [8], but good spatial separation of the ion beam from the neutral component requires rather high voltages on the plates because of the significant divergence of the beam. Operating as an ion deflector, the EPG produces a cylindrically symmetric deflection pattern for transmitted ions, and the trajec- 
tories of the neutral components remain unperturbed. In this study, the action of an EPG used as an ion deflector in both continuous and pulsed modes is examined. Examples are given of its use in measuring the neutral component of a mass spectrum and in reducing background by acting as an electrostatic ion gate.

\section{Experimental}

\section{Instrumentation}

A linear PDMS configuration was used in this study, the basic details of which are given elsewhere [9]. The specific arrangement employed is shown schematically in Figure 1. The ${ }^{252} \mathrm{Cf}$ source was situated in the $3 \mathrm{~mm}$ of space between the fission event detector (start detector) and the target position. The secondary ion detector (stop detector) was located at the end of the desorbed ion flight path. Each detector consisted of two microchannel plates (MCP) (Galileo Electro-optics, Galileo Park, Sturbridge, MA) in a chevron configuration. The stop detector was mounted on a linear motion feedthrough device such that the flight path was variable with a range of $32-52 \mathrm{~cm}$. An electron-focusing ring was positioned between the stop detector MCP chevron and the impedance-matched conical anode in order to collect electrons from the entire $40-\mathrm{mm}$ active diameter of the MCPs on the $20-\mathrm{mm}$ diameter anode [10]. A variable-diameter $(4-40 \mathrm{~mm})$ iris diaphragm (Edmund Scientific, Barrington, NJ) was placed in an axial location $2 \mathrm{~cm}$ in front of the stop detector face. Control of the aperture size was external, via a push-pull linear motion feedthrough. A fixed collimator, $36 \mathrm{~mm}$ in diameter, was placed $5 \mathrm{~mm}$ downstream of the EPG. The EPG was located in the field-free region of the ion flight path, $74 \mathrm{~mm}$ from the acceler- ation grid. It was constructed from a stainless steel cylinder $10 \mathrm{~cm}$ long and $40 \mathrm{~mm}$ in diameter. A $0.173-$ mm-diameter stainless steel wire was suspended along the axis of the cylinder and supported at each end by a wire mounted across the middle of the cylinder apertures, perpendicular to the center wire (Figure 1). The wires were electrically insulated from the grounded cylinder and were connected to a high-voltage supply through one of the support wires.

Fast preamplifiers (Ortec 9301) were used to amplify the signals from the start and stop detectors. The signals were then processed by Canberra Model $1428 \mathrm{~A}$ constant-fraction discriminators and sent to a custom-built multistop time digitizer [11]. The output of the digitizer was interfaced to a Concurrent 3210 computer, which was used for data acquisition and analysis [9].

The reverse EPG can be operated in an on/off mode or in a pulsed mode [7]. In the on/off mode the entire spectrum is collected with the reverse EPG voltage either on or off. When the EPG is on, all ions are deflected to such an extent that they are not detected at the end of the flight tube, which means that the recorded spectrum represents the neutral component. When the EPG is off, the entire spectrum (ions and neutrals) is recorded. Intermediate EPG voltages result in a spatial separation of ions from neutral fragments, but both are still detected. This makes it possible to record spectra of neutral fragments and ion beam profiles at different reverse EPG voltages. The pulsed mode reverse EPG is a method for minimizing the intensity of the uncorrelated background by introducing constraints to the range of acceptable time-of-flight values [8]. The schematic of the EPG pulser circuitry is given in Figure 2. In a pulsed off mode, voltage is applied to the EPG continuously but is "pulsed off" for a

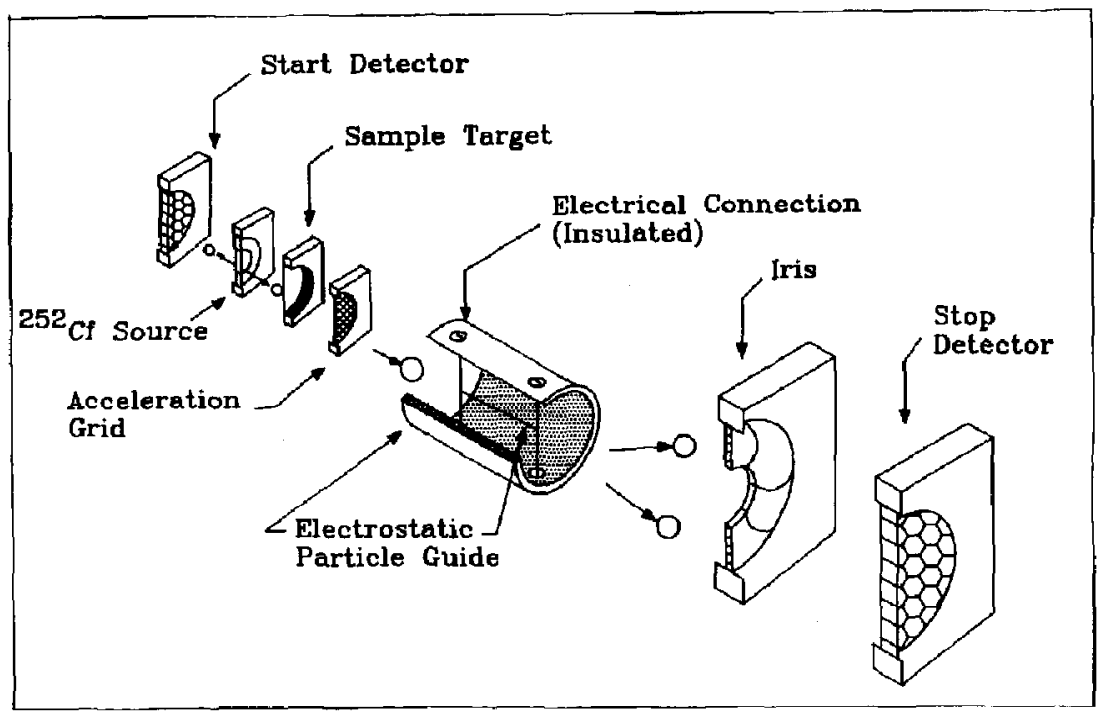

Figure 1. Isometric cutaway view of the ${ }^{252} \mathrm{CF}-\mathrm{PDMS}$ using the EPG in the deflection mode. 


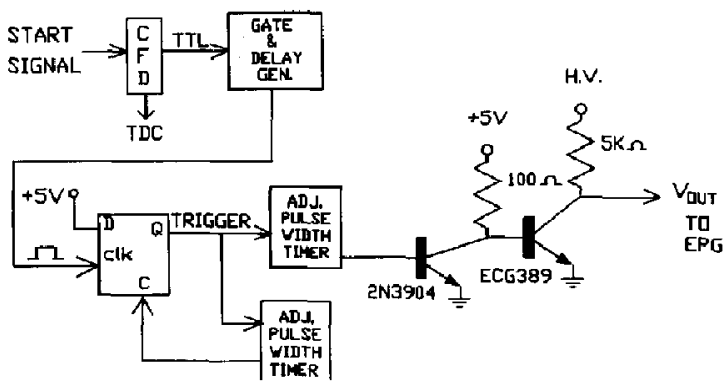

Figure 2. Schematic of the EPG pulser circuitry.

time interval corresponding to the acceptable range of time-of-flight values. When a start signal is received, the EPG voltage remains on for a delay period and then is switched off for the duration of a gated period. Only those ions passing through the EPG during the gated period will be detected. Delay time and gate width are chosen according to estimates of locations of the desired time packet of the ions in the flight path. In this study, the gate and delay were set such that the EPG deflecting voltage was switched off just before the quasi-molecular ion entered the EPG. The voltage remained off for a period corresponding to the time spent by the ion in traversing the length of the EPG and was then switched back on. These times were calculated on the basis of acceleration voltage, distance to the EPG from the target, length of the EPG, and desired mass range for the transmitted ions.

\section{Principles and Simulations}

The principles of operation of the reverse EPG are essentially the same as for the EPG in the standard mode, but the polarity of the reverse EPG voltage is the same as that of the accelerated secondary ions, so the ions are deflected from the axis of the EPG. The electric field inside the EPG increases the radial component of velocity of the ions traversing through it. Therefore, the time of flight of the ions should not be perturbed, except by fringe electrostatic fields at the ends of the EPG [7].

An electrostatics simulation program, SIMION [12], was used to model the deflection system with the reverse EPG at various voltages. The trajectories were calculated for desorption from a 6-mm-diameter target area with $5 \mathrm{eV}$ initial energy [13]. Ions were simulated as being emitted from the target at angles ranging from $-90^{\circ}$ to $+90^{\circ}$ in steps of $30^{\circ}$ from an axis centered along the flight path. Acceleration voltage was set at $+15 \mathrm{kV}$ for the simulations. Results of the simulations are shown in Figure 3. The program used cylindrical symmetry for the simulations, but the resulting plots are cross-sectional views. Positive ion trajectories with the reverse EPG off are shown in Figure 3a. The ions traverse the flight path to the stop detector, diverg-

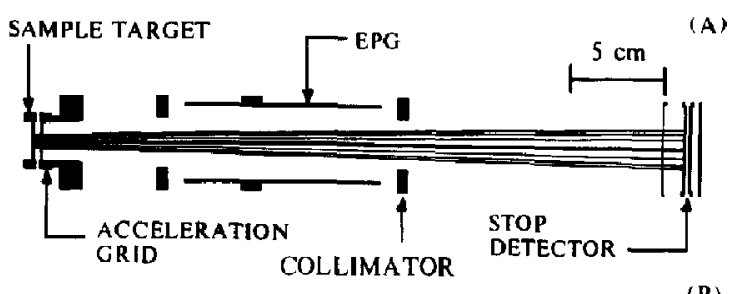

(B)

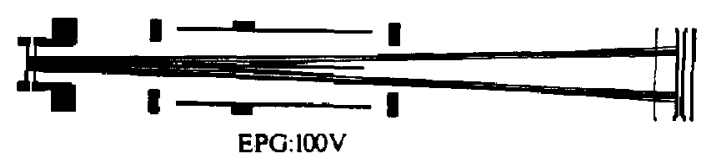

(C)

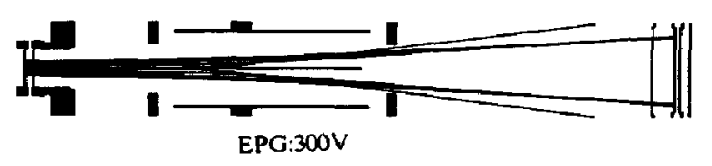

(D)
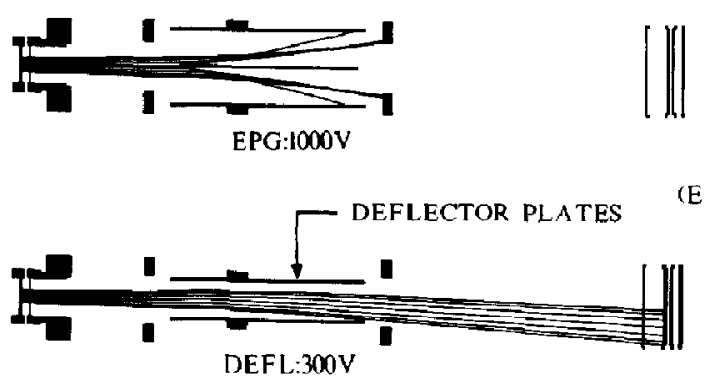

Figure 3. Two-dimensional illustrations of SIMION calculated trajectories for ions traversing the spectrometer with EPG deflection voltages of (a) $0 \mathrm{~V}$, (b) $100 \mathrm{~V}$, (c) $300 \mathrm{~V}$, and (d) $1000 \mathrm{~V}$. (e) A SIMION calculation for $300 \mathrm{~V}$ across two deflector plates.

ing to a diameter of about $25 \mathrm{~mm}$ at the location of the stop detector. Figure $3 \mathrm{~b}$ and $\mathrm{c}$ show the same trajectories for $+100 \mathrm{~V}$ and $+300 \mathrm{~V}$ on the EPG, where the ions are deflected from the axis but still strike the detector. Figure 3d shows the same set of trajectories with the reverse EPG set at $+1000 \mathrm{~V}$. At this voltage, all ions are deflected into an annulus with an inner diameter larger than the diameter of the collimator (36 $\mathrm{mm}$ ) behind the EPG. Note that the closer the ions are to the EPG wire, the more they are deflected, because the field strength is highest near the center wire. Under these conditions, the only events recorded are due to energetic neutral species produced by metastable decay during and after ion acceleration. For comparison, SIMION calculations were performed for deflector plates under the same conditions as for the EPG. The distance between the plates corresponds to the radius of the EPG, and the length of the plates is identical to the EPG. It should be noted that the noncylindri- 
cal lens elements (the parallel plates) preclude the use of cylindrical symmetry in the simulations. Trajectories were calculated for ions using the same conditions as in the EPG simulations. The simulation for $+300 \mathrm{~V}$ across the deflector plates (bottom plate at ground and top plate at $+300 \mathrm{~V}$ ) is shown in Figure 3e. The simulations clearly show that for the same applied potential, ions are not as greatly deflected with the parallel-plate configuration as with the EPG under the stated conditions. Geometry is an important factor in the superiority of the EPG deflection over parallel plates. In the EPG, electric field strength is great in the immediate vicinity of the center wire but rapidly decreases with distance from the wire. The parallel deflector plates provide a strong electric field that is more uniformly distributed between the two plates. The geometry of the ion beam, however, is such that most of the ions will travel in the region close to the EPG center wire, whereas many of the ions traveling through the deflector plates will be in the regions of weaker field strength. In addition, the force exerted on the ions in the EPG is always in the same direction as the initial radial velocity vectors of those ions. As shown in Figure $3 e$, half of the ions traversing the parallel plates are traveling toward the deflection field, so that much of the deflection power is lost in reversing the radial direction of these ions. The net effect is that more voltage is required on the parallel plates to achieve a deflection comparable to that of the EPG.

\section{Sample Preparation}

For these studies, targets were prepared by adsorbing sample from solution onto an electrosprayed nitrocellulose matrix on an aluminized mylar film [14]. Details of the electrospraying procedure are described elsewhere [15]. The sample used in these studies was bovine insulin (5734 u) obtained from Sigma Chemical (St. Louis, MO). The bovine insulin was dissolved in a solvent consisting of $0.1 \%$ aqueous trifluoroacetic acid and ethanol (50:50) to give a $1 \mathrm{mM}$ solution. Ammonium hydroxide was used to adjust the solution to $\mathrm{pH} \approx 5$ before adsorption on the nitrocellulose. These conditions were found to give optimal molecular ion yields.

\section{Measurement Protocol}

A general procedure for the measurement of ion beam profiles was developed for this study. An iris diaphragm was positioned directly in front of the stop detector and on the axis of the spectrometer. The iris diameter was externally controlled by a push-pull linear motion feedthrough. The iris aperture diameters were calibrated to correspond to different lengths of the push-pull rod. Aluminum spacers of various lengths were used to hold the push-pull rod in place. Repeatability of the aperture diameters was found to

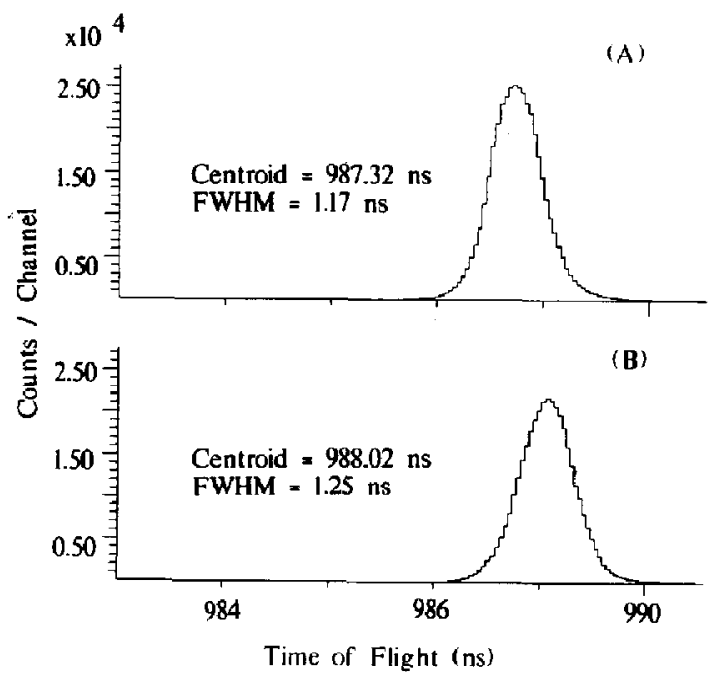

Figure 4. Hydrogen ion spectra recorded at 78 ps per channel with (a) no voltage and (b) $+300 \mathrm{~V}$ deflection on the EPG.

be within $\pm 0.3 \mathrm{~mm}$. Ten iris diameters ranging from 4.1 to $40.0 \mathrm{~mm}$ were chosen for measuring profiles. Spectra were acquired for $5 \mathrm{~min}$ at each of the ten diameters for each profile. The primary acceleration voltage used in these acquisitions was $+15 \mathrm{kV}$, and a flight path of $32 \mathrm{~cm}$ was employed.

\section{Results and Discussion}

\section{Effect on Calibration Ions}

Our initial concern in this study was to determine the degree of distortion of the time-of-flight spectra due to acceleration/deceleration by the fringe electrostatic fields at the end of the EPG. Pronounced effects were observed in previous studies for low-energy ions [7]. Positive ion spectra of a bovine insulin sample (MW = 5734) were recorded at each of six EPG voltages, ranging from 0 to $+500 \mathrm{~V}$, and at an acceleration voltage of $+15 \mathrm{kV}$. Figure 4 shows the $\mathrm{H}^{+}$calibration ton region of a spectrum recorded at 78 ps per channel with no voltage on the EPG (Figure 4a), and with a deflection voltage of $+300 \mathrm{~V}$ (Figure $4 \mathrm{~b}$ ). This deflection voltage setting was found to impart the optimum divergence of the beam without a great loss of intensity at the end of the flight tube. At an EPG voltage of $+300 \mathrm{~V}$, the centroid of the $\mathrm{H}^{+}$peak is shifted to a slightly longer time of flight from that of the EPG at $0 \mathrm{~V}$ due to electrostatic repulsion in the fringe field region; however, the peak shape is undistorted. This means that the mass calibration is changed when the EPG is activated, but it is possible to obtain a new calibration curve from the shifted time-of-flight spectra of the calibration ions. 
Figure 5. Background-subtracted molecular ion regions from positive ion spectra of bovine insulin recorded with (a) no voltage and (b) $+3 m \mathrm{~V}$ deflection on the EPG.

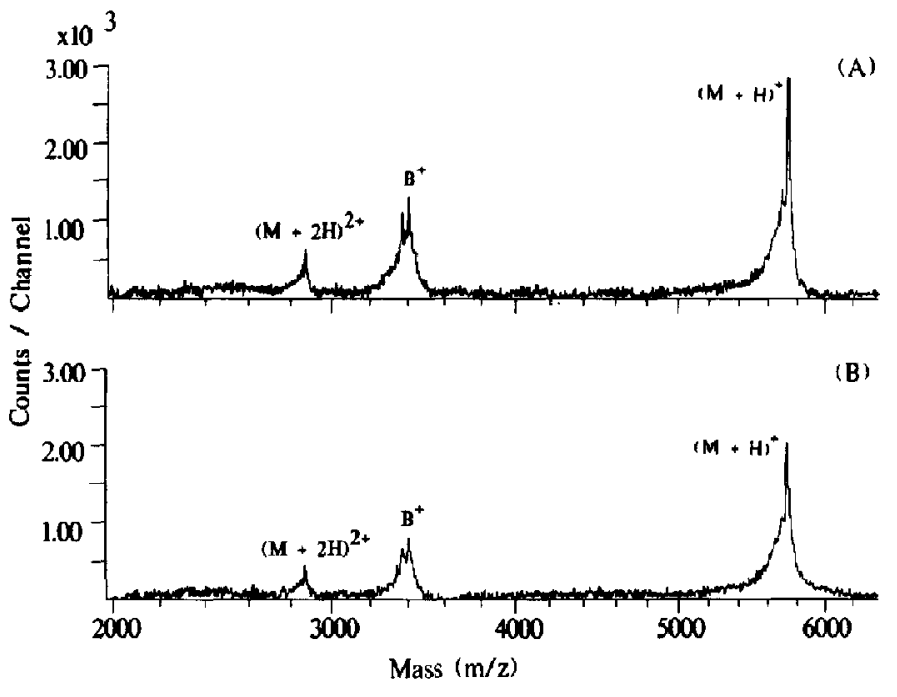

\section{Effect on Mass Spectra}

The influence of the action of the EPG on the molecular ion region of insulin is shown in Figure 5. Figure $5 \mathrm{a}$ is recorded with no EPG voltage, and Figure $5 b$ with an EPG voltage of $+300 \mathrm{~V}$. The smooth background for each spectrum has been subtracted for this figure. The prominent peaks in the spectrum are due to the molecular ion at $m / z 5735$, the $2+$ ion at $m / z 2868$, and an ion from the $B$ chain. The influence of the EPG has two effects: It shifts the time-of-flight spectrum to higher times as observed with the $\mathrm{H}^{+}$ion, and the intensity is reduced to $70 \%$ of its original value. This is due to deflection of a component of the ion beam to a radius that exceeds the size of the $40-\mathrm{mm}$-diameter MCP ion detector. The pattern of deflected ions is discussed in detail in the next section.

\section{Measurement of Radial Distribution Functions of Ions for Different Electrostatic Particle Guide Deflection Voltages}

The objective of this part of the study was to determine how the EPG deflection system influenced the spatial distribution of the ions at the ion detector surface at the end of the flight tube. The final form of the data is presented as a radial distribution function that gives the probability of an ion of a given mass being detected within a concentric ring with a fixed width as a function of radius.

The measurement protocol is outlined in the experimental section. Two ions in the mass spectrum of bovine insulin deposited on nitrocellulose were used in this study. One of these, the $\mathrm{H}^{+}$ion, is representative of the class of ions that has a relatively large radial velocity component [16]. The other ion, $\mathrm{NO}^{+}$, has a much smaller divergence. The differences in the interaction of these ions with the EPG deflector give information on the role of the initial radial velocity distribution in influencing the radial distribution functions for different EPG voltages.

Mass spectra were recorded for a set of EPG voltages in the range of 0 to $+500 \mathrm{~V}$, and integrated intensities were measured for the two ions. The results for $\mathrm{H}^{+}$plotted as intensity (relative to intensity when the iris is fully open and without EPG voltage) versus iris diameter are shown in Figure 6. There are several general features of the data worth noting. First, the curve with an EPG voltage of zero represents the unperturbed beam profile for the $\mathrm{H}^{+}$ion. The integrated intensity reaches a maximum value at a $30-\mathrm{mm}$ diameter iris aperture with a 32-cm flight path and $+15-\mathrm{kV}$ acceleration. This means that the ion detec-

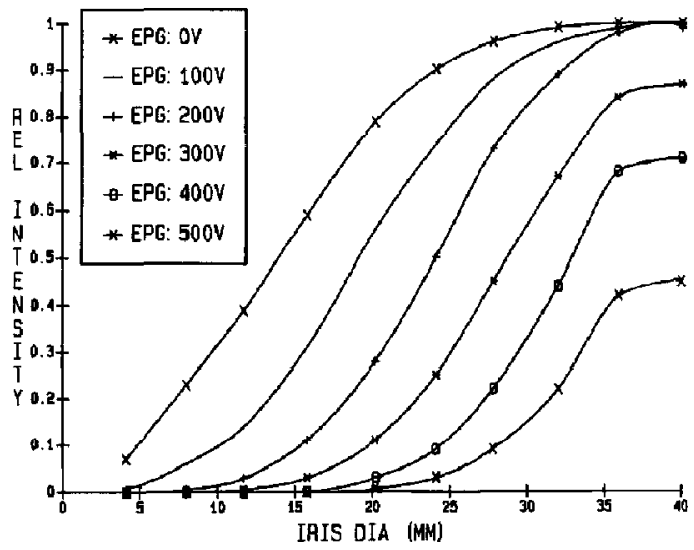

Figure 6. Intensity of the $\mathrm{H}^{+}$peak plotted as a function of iris diameter for various EPG deflection voltages. Intensity values are relative to the $\mathrm{H}^{+}$intensity with the EPG at $0 \mathrm{~V}$ and with an iris diameter of $40 \mathrm{~mm}$. 
tor area is larger than the area of the unperturbed ion beam, even for the most divergent ion. In the EPG voltage range of $+100-200 \mathrm{~V}$, the ion intensities for the smaller iris apertures are diminished with increasing voltage, but the intensities at full aperture opening remain constant. This means that the ions are being deflected to larger radii but are still within the active area of the ion detector. At an EPG voltage greater than $+200 \mathrm{~V}$, there is a loss of $\mathrm{H}^{+}$intensity due to deflection of a component of the ion beam to radial displacements exceeding the diameter of the ion detector. At the highest EPG voltage $(+500 \mathrm{~V})$, the $\mathrm{H}^{+}$ion intensity within a $20-\mathrm{mm}$ diameter of the spectrometer axis at the end of the flight tube is totally depleted, and approximately $60 \%$ of the ions are deflected beyond the active diameter of the ion detector. From the beam profile data, the qualitative action of the EPG can be deduced. The EPG converts the distribution from a disk to a ring with an inner and outer diameter that become larger with increasing EPG voltage. Approximations of the inner diameter of the deflected beam can be made by determining the iris radius corresponding to the inflection point of the profile curve for various EPG voltages.

Radial distribution function plots were derived from the integrated beam profile data shown in Figure 6 . The transformation of the beam profile data to radial distribution function plots was done in the following manner for each EPG voltage. First, the intensity for a particular annular ring as a function of radius was calculated by subtracting the measured intensity for a given iris setting from that observed for an iris setting that was slightly larger. The resulting "annular intensities" were divided by the maximum intensity observed with the iris fully open. This procedure is essentially equivalent to differentiating the measured beam profile shown in Figure 6.

The radial distribution functions at EPG deflection voltages of $0 \mathrm{~V},+100 \mathrm{~V},+300 \mathrm{~V}$, and $+400 \mathrm{~V}$ are shown in Figures 7 and 8 for $\mathrm{H}^{+}$and $\mathrm{NO}^{+}$, respectively. These plots show the spatial distributions of the ions as they strike the detector at the end of the flight path. Both figures show that the ions are increasingly deflected with increasing EPG potential. The distributions also show the divergence of $\mathrm{H}^{+}$to be greater than that of $\mathrm{NO}^{+}$at each of the deflection voltages. The spread of the $\mathrm{H}^{+}$distribution functions is larger than for $\mathrm{NO}^{+}$. This means that the spread of initial radial velocities of $\mathrm{H}^{+}$ions is greater than that of $\mathrm{NO}^{+}$ ions, as one would expect. The plots are also an important indication of the area that is essentially free of ions at each EPG voltage. For example, at $+300 \mathrm{~V}$. most of the ions are located outside a $10-\mathrm{mm}$ radius. From the probability densities, one can directly calculate the fraction of ions outside a particular radius for a deflection voltage. This provides an explicit way to determine the minimum EPG voltage required to deflect all of the ions out of a given circular area around the axis of the spectrometer. When the excluded area is

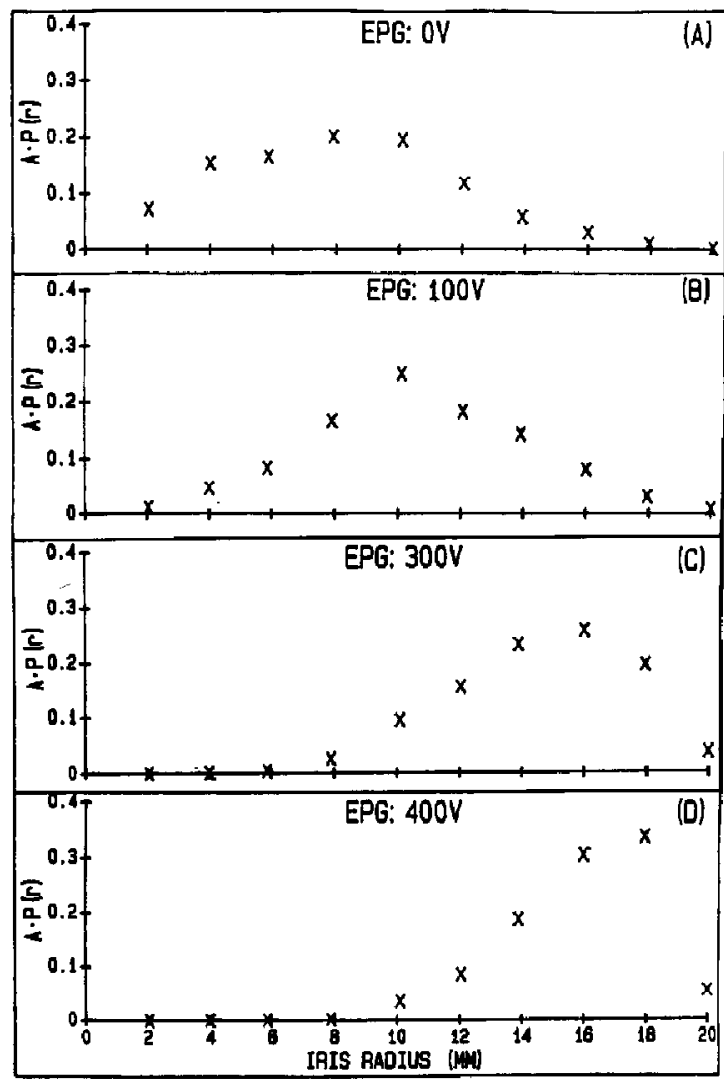

Figure 7. Radial distribution function for $\mathrm{H}^{+}$with EPG deflection voltages of (a) $0 \mathrm{~V}$, (b) $+100 \mathrm{~V}$, (c) $+300 \mathrm{~V}$, and (d) +400 V. $P(r)$ is the probability density, and $A$ is the annular area.

larger than the area of the ion detector for a given deflection voltage, only the energetic neutral component of the particle beam will be detected. Spectra recorded with this deflection voltage will show the neutral component only.

\section{Action of Deflection Electrostatic Particle Guide}

Plots of ion intensities versus EPG deflection voltage (EPG action curves) show the influence on ion transmission of the deflection EPG as a function of its voltage. The EPG action curves for flight lengths of 32 $\mathrm{cm}$ and $52 \mathrm{~cm}$ with the iris fully open are displayed in Figure 9. Ion deflection beyond the diameter of the ion detector is essentially complete at an EPG voltage of $+600 \mathrm{~V}$ for the $52-\mathrm{cm}$ flight length and at +1000 $\mathrm{V}$ for the $32-\mathrm{cm}$ flight length. The voltage required to completely deflect the ions is less for the longer flight length because the deflected ions have more time to spread radially before reaching the detector.

\section{Neutral Species Spectra}

Figure 10 shows the molecular ion region of an insulin spectrum acquired with the deflection EPG voltage at 


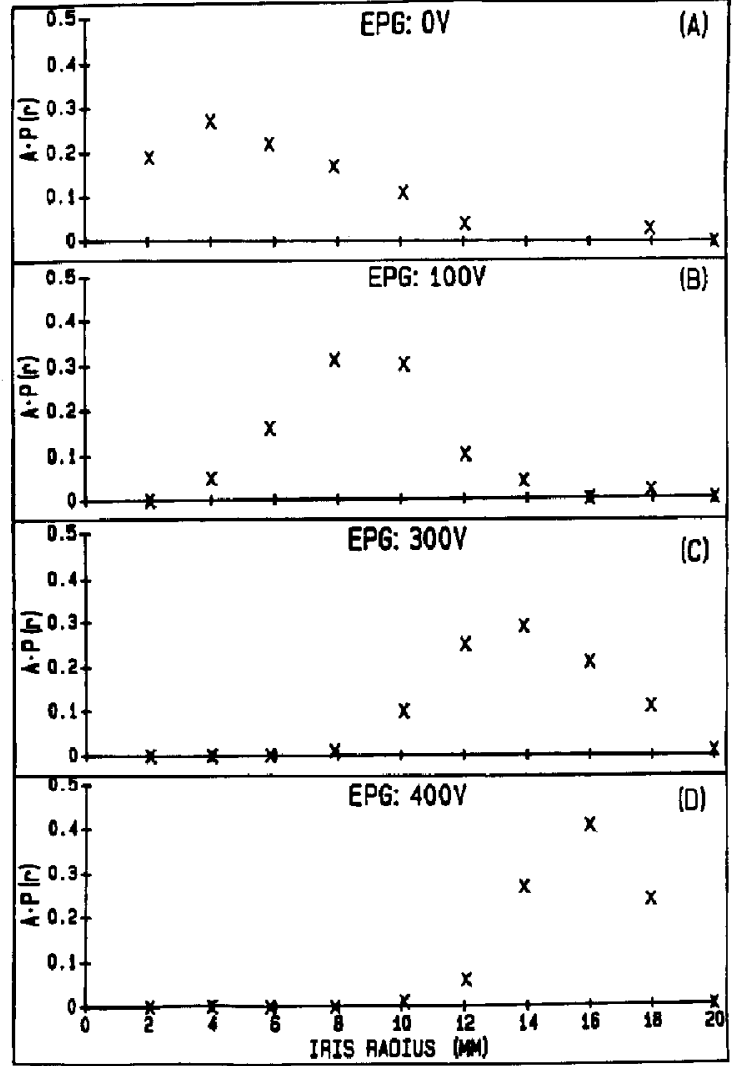

Figure 8. Radial distribution function for $\mathrm{NO}^{+}$with EPG deflection voltages of (a) $0 \mathrm{~V}$, (b) $+100 \mathrm{~V}$, (c) $+300 \mathrm{~V}$, and (d) +400 V. $P(r)$ is the probability density, and $A$ is the annular area.

zero potential (a) and with the EPG on at $+1000 \mathrm{~V}(\mathrm{~b})$ in on/off mode. The spectrum in Figure $10 \mathrm{~b}$ constitutes a neutral species spectrum because all the ions have been deflected from the ion detector. The subtraction of the Figure $10 \mathrm{~b}$ spectrum from the Figure $9 \mathrm{a}$ spectrum yields the spectrum shown in Figure 10c. The neutral species that were detected were formed from metastable ions that dissociated in a manner such that the resulting neutral species had sufficient velocity to be detected. It is apparent from Figure 10a that approximately $35 \%$ of the background in the high-mass region $(m / z 6000)$ is due to energetic neutral species. Subtraction of the neutral species spectrum from the total spectrum (ions + neutral fragments) essentially eliminates the slope in the background spectrum.

The energetic neutral molecule spectra from metastable decay can also provide information about the fragmentation processes that occur. For example, decays that yield neutral fragments from positive ions differ from those of negative ions. This is illustrated in Figure 11, in which an energetic neutral molecule spectrum resulting from positive ion decay (a) is compared with the corresponding spectrum from negative ion decay (b) for the redox polymer polypyrrole with

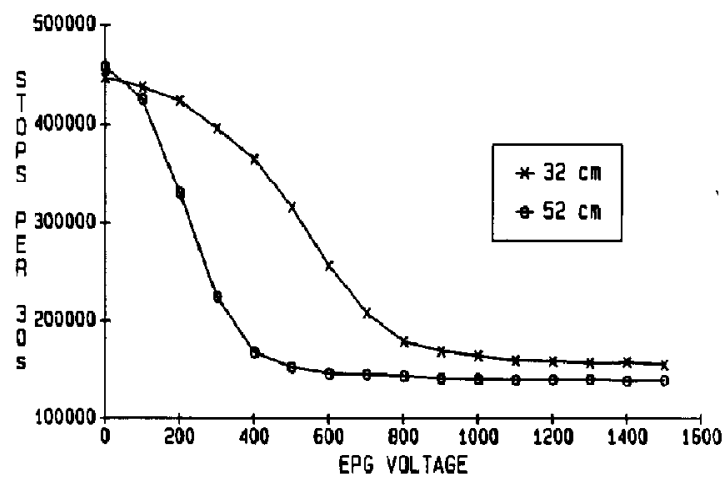

Figure 9. The EPG action curves for flight lengths of $32 \mathrm{~cm}$ and $52 \mathrm{~cm}$. The counting rates above $1000 \mathrm{~V}$ represent the neutral component of the spectrum.

the counterion sulforhodamine B. The peaks shown in the "negative" neutral spectrum are much sharper than those in the "positive" neutral spectrum. An explanation for this involves different neutralization processes for the two cases. In the case of negative ions, neutral fragments can be formed by the loss of an electron. In order to neutralize a positive ion, the loss of an $\mathrm{H}^{+}$or a larger positive fragment is required. When fragmentation occurs, the internal energy of excitation of the precursor ion is converted into kinetic energy

$$
\times 10^{3}
$$
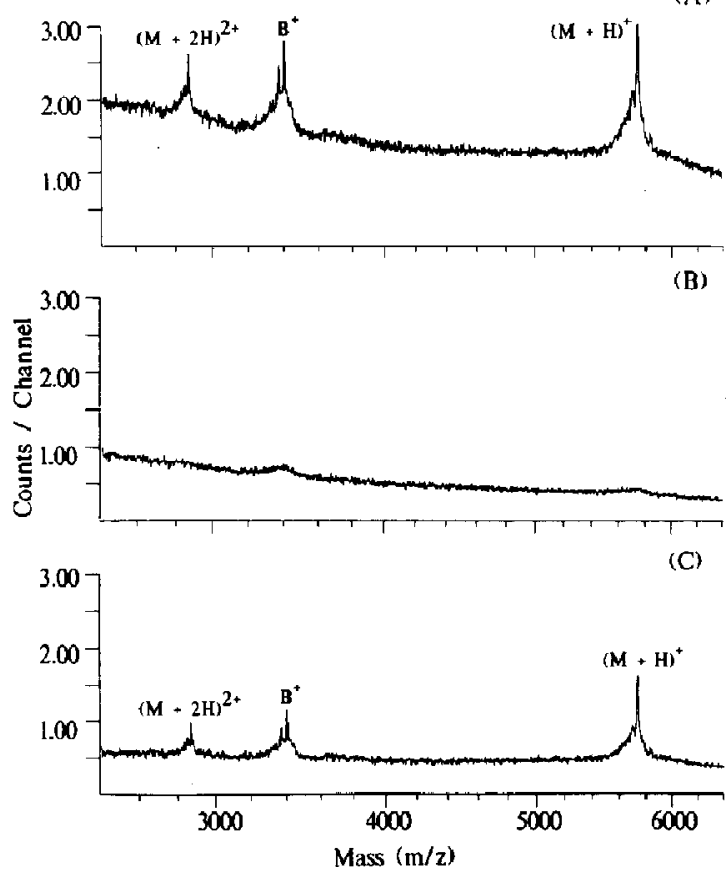

Figure 10. Molecular ion regions of bovine insulin spectra acquired with (a) $0 \mathrm{~V}$ and (b) $+1000 \mathrm{~V}$ EPG deflection voltage. The spectrum shown in (c) is the result of subtracting spectrum (b), the neutrals spectrum, from spectrum (a) and represents the ionic component of the spectrum. 


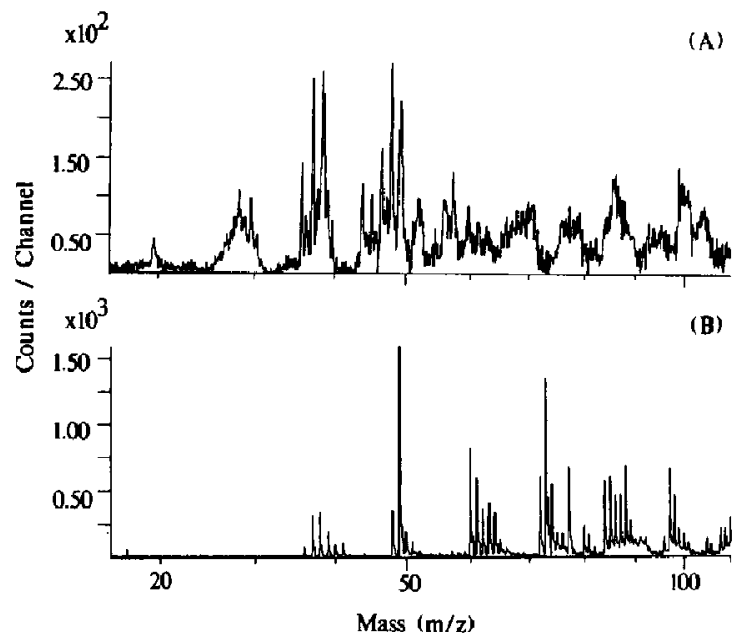

Figure 11. Partial spectra of neutrais resulting from (a) positive ion decay and (b) negative ion decay for a typical sample. Spectra were recorded with a deflection voltage of $+1000 \mathrm{~V}$.

of the fragments. The resulting velocities of the fragments relative to the precursor are inversely proportional to their masses as a consequence of conservation of momentum. The neutral fragment velocities are distributed about the mean velocity of the precursor, resulting in a broadened peak in the spectrum. The degree of peak broadening is proportional to the ratio of the mass of the precursor ion to that of the neutral fragment. Neutral fragments resulting from the loss of an electron would have essentially the same mass as the precursor ion, and therefore peak broadening would be extremely small for these neutrals compared to the loss of a proton or heavier ionic species.

\section{Pulsed Mode Deflection Electrostatic Particle Guide}

Some results of the pulsed EPG experiments are compared in Figure 12. The deflection voltage applied to the EPG for the pulsed experiments was $+800 \mathrm{~V}$, a value that was found to deflect the ions completely out of the detection area. Figures 12a and 13a show the low- and high-mass regions, respectively, of insulin spectra with the EPG pulser turned off. Figures $12 b$ and $c$ and $13 b$ and $c$ demonstrate the effects of different pulser windows on the spectra. The case shown in Figures $12 b$ and $13 b$ is for a window with a delay of $0.5 \mu s$ and a width of $0.5 \mu$ s (i.e., the deflection EPG is "pulsed off" for $0.5 \mu \mathrm{s}$ after a delay period of $0.5 \mu \mathrm{s}$ ). Only those ions traversing through the EPG within the time period defined by the pulser window can be detected. Under these conditions, only a narrow range of ions is detected, those from $m / z 30$ to $m / z 150$. In the region of the molecular ion of insulin, only the neutral background is present, as shown in Figure $13 \mathrm{~b}$. The minimum delay period is limited by the pulser circuitry and was estimated to be $0.5 \mu \mathrm{s}$. As shown in Figure 12b, the deflection voltage could not be turned
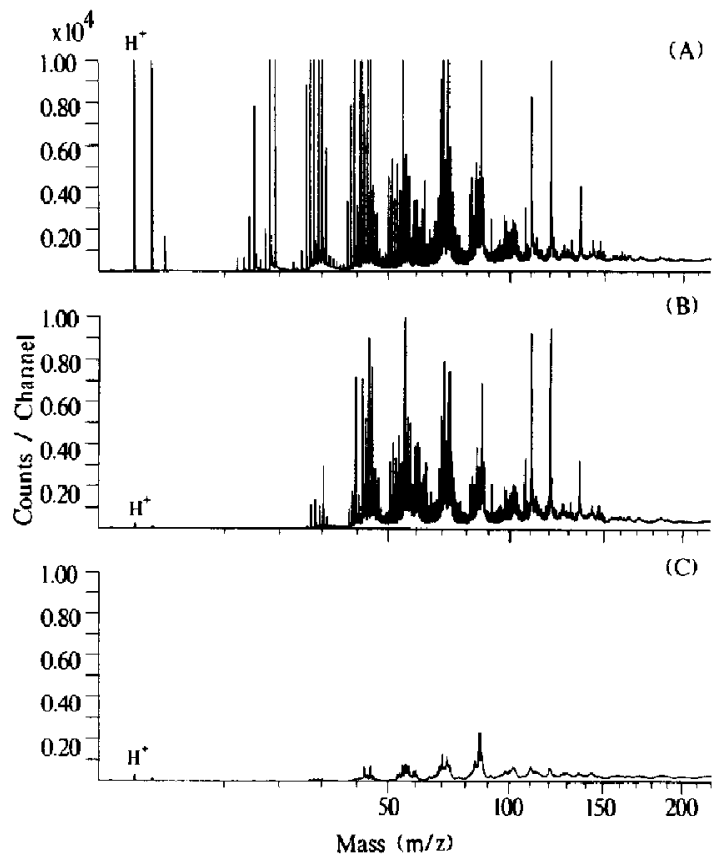

Figure 12. Low-mass regions of a bovine insulin sample with (a) the EPG pulser turned off, (b) the EPG pulser on with a delay of $0.5 \mu \mathrm{s}$ and a width of $0.5 \mu \mathrm{s}$, which transmits ions in the mass range $m / z 30-150$, and (c) the EPG pulser on with a delay of 5.0 $\mu \mathrm{s}$ and $a$ width of $6.0 \mu \mathrm{s}$, which transmits ions in the mass range $m / z$ 2000-6000. Note that many of the peaks in (a) are off scale. The residual spectrum (c) is the neutral component.

off fast enough to include $\mathrm{H}^{+}$in the low-mass window of detected ions. Figures $12 c$ and $13 c$ show the spectra acquired with a pulser delay of $5.0 \mu \mathrm{s}$ and a width of $6.0 \mu \mathrm{s}$. This window allows those jons in the mass region of the molecular ion of insulin to be transmitted undeflected through the EPG. Ions arriving before and after this time window are deflected beyond the acceptance cone of the ion detector. The intensity of the $\mathrm{H}^{\prime}$, for example, is reduced to less than $1 \%$ of its original value (Figure 12a).

\section{Summary}

An EPG operating with a reversed polarity is described and is shown to be effective for deflecting a divergent beam of ions into a pattern with cylindrical geometry. This technique provides a cleaner method for ion deflection than the standard parallel-plate deflection method for two reasons. First, the force exerted on the ions is much greater due to the large field in the region of the central wire, and, second, it is always in the same direction as the initial radial velocity vector of the ion. The device, operating in the on/off mode at high voltage, can be used for large-angle ion deflection to study the neutral component of the particle beam. At intermediate voltages, it would be possible to separate ions from neutral fragments by deflecting 

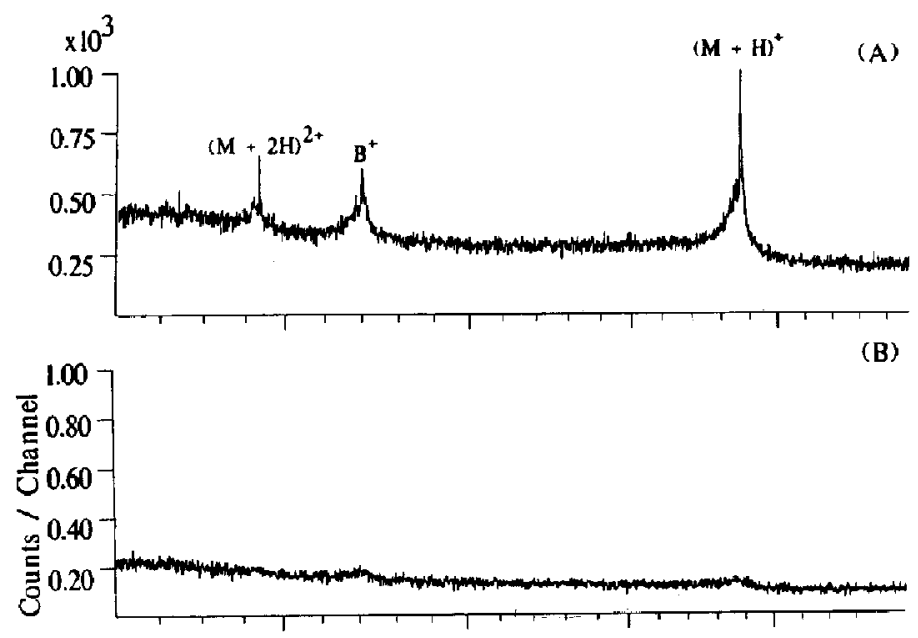

B)

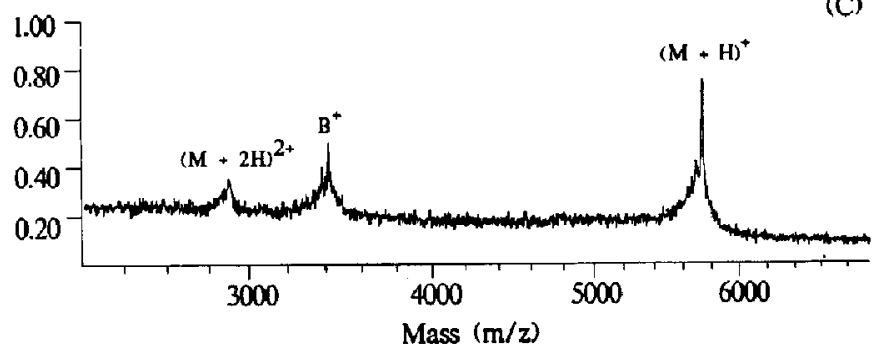

Figure 13. Molecular ion regions of bovine insulin spectra with (a) the EPG pulser turned off, (b) the EPG pulser on with a delay of $0.5 \mu \mathrm{s}$ and a width of $0.5 \mu \mathrm{s}$, and (c) the EPG pulser on with a delay of $5.0 \mu \mathrm{s}$ and a width of $6.0 \mu \mathrm{s}$. The transmitted mass ranges are the same as for Figure 12.

the ions into a cylindrical ring within the active area of a ion detector and shielding the central region of the detector where the neutral component of the beam is concentrated. Radial distribution functions have been measured for $\mathrm{H}^{+}$, an ion with a high radial velocity distribution, and for $\mathrm{NO}^{+}$, an ion with a small radial velocity distribution. These distributions show how the radius of the cylindrical ring increases with increasing EPG voltage. Finally, operating in a pulsed mode synchronized with the nuclear fission event characteristic of ${ }^{252}$ Cf-PDMS, the device is shown to be an effective electrostatic gate for transmitting ions within a particular time-of-flight interval, providing a means for reducing the background in the mass spectrum.

\section{Acknowledgments}

We wish to thank Dennis Shelton for significant technological contributions to this study. This research was sponsored by the National Science Foundation (CHE-8604609), the National Institutes of Health (GM-26096), and the Robert A. Welch Foundation (A-258).

\section{References}

1. Macfarlane, R. D.; Sundqvist, B. U. R. Mass Spectrom. Rev. $1985,4,421-460$.

2. Craig, A. G.; Engstrom, A.; Bennich, H.; Kamensky, I. Presented at the 35th ASMS Conference on Mass Spectrometry and Allied Topics, Denver, CO, May 23-29, 1987.
3. Chait, B. T.; Field, F. H. Int. J. Mass Spectrom. Ion Phys. 1981, $41,17-29$.

4. Sundqvist, B. U. R.; Kamensky, I.; Hakansson, P.; Kjellberg, J.; Salehpour, M.; Widdiyasekera, S.; Fohlman, J.; Peterson, P. A.; Roepstorff, P. Biomedical Mass Spectrometry 1984, 11. 242-257.

5. Macfarlane, R. D.; Torgerson, D. F. Int. J. Mass Spectrom. Ion Phys. 1976, 21, 81-92.

6. Oakey, N. S.; Macfarlane, R. D. Nucl. Instrum. Methods 1967, 49, 220-228.

7. Geno, P. W.; Macfarlane, R. D. Int. I. Mass Spectrom. Ion Phys. 1986, 74, 43-57.

8. Hedin, A.; Hakansson, P.; Sundqvist, B. U. R. Int. J. Mass Spectrom. Ion Phys. 1986, 70, 203-212.

9. Macfarlane, R. D. Anal. Chem. 1983, 55, 1247A-1263A.

10. P. D. Mudgett and R. D. Macfarlane, unpublished results.

11. Turko, B. T.; Macfarlane, R. D.; McNeal, C. J. Int. J. Mass Spectrom. Ion Phys. 1983, 53, 353-362.

12. Dahl, D. A.; Delmore, J. E. SIMION, version 4.0, EGG-CS7233, EG\& G Idaho, Idaho National Engineering Laboratory, Idaho Falls, ID, 1988.

13. Macfarlane, R. D.; Hill, J. C.; Jacobs, D. L. J. Trace Microprobe Tech. 1986-87, 4, 281-302.

14. Jonsson, G. P.; Hedin, A. B.; Hakansson, P. L.; Sundqvist, B. U. R.; Save, B. G. S.; Nilsen, P. F.; Roepstorff, P.; Johansson, K.; Kamensky, I.; Lindberg, M. S. L. Anal. Chem. 1986, 58, 1084-1087.

15. McNeal, C. J.; Macfarlane, R. D.; Thurston, E. L. Anal. Chem. 1979, 51, 2036-2039.

16. Mudgett, P. D.; Macfarlane, R. D. Proceedings of the 5th IFOS Conference, Lovanger, Sweden, June 1989 (in press). 